\title{
Transcatheter Aortic Valve Replacement in Female Patients-Present Scenario
}

\author{
Osnat Itzhaki Ben Zadok ${ }^{1}$ Katia Orvin ${ }^{1}$ Ran Kornowski ${ }^{1}$ \\ ${ }^{1}$ Department of Cardiology, Rabin Medical Center, and Sackler \\ Faculty of Medicine, Tel Aviv University, Petah Tikva, Israel \\ Indian J Cardiovasc Dis Women-WINCARS 2017;2:7-13.

\begin{abstract}
Address for correspondence Ran Kornowski, MD, FESC, FACC Division of Cardiovascular Medicine, Department of Cardiology, Rabin Medical Center, Tel Aviv University, Beilinson and Hasharon Hospitals, Petah Tikva, Israel 4941492 (e-mail: ran.kornowski@gmail.com).
\end{abstract}

\begin{abstract}
Keywords

- aortic stenosis

- PARTNER trial

- surgical aortic valve replacement

- transcatheter aortic valve replacement

Since the publication of the pivotal PARTNER study, the transcatheter aortic valve replacement (TAVR) procedure has been established as a noninferior alternative to the traditional aortic valve replacement surgery in severe aortic stenosis (AS) patients with high-surgical risk. Approximately $50 \%$ of patients undergoing TAVR are females, and cumulative findings from various worldwide cohorts have shown sex-related differences in short- and long-term morbidity and mortality. Notably, most data indicate improved long-term mortality in female patients. These differences are partly the results of distinct anatomical and physiologic characteristics in female patients, compared with male patients. Nevertheless, recent data from intermediate-surgical risk cohorts have demonstrated that sex-related mortality differences are less apparent. Here, we review the latest literature on the influence of patient's sex on TAVR morbidity and mortality and discuss possible explanations for the outcomes presented.
\end{abstract}

As the prevalence of severe symptomatic aortic stenosis (AS) increases with age, the use of transcatheter aortic valve replacement (TAVR) is expanding as an acceptable alternative to the traditional surgical aortic valve replacement (SAVR) in intermediate- and high-surgical risk patients. ${ }^{1-4}$ Based on several worldwide registries, approximately $50 \%$ of patients undergoing TAVR are females, ${ }^{5-7}$ and compared with male patients, they carry distinct anatomical and physiologic characteristics and subject to different shortand long-term morbidity and mortality.

Female patients with severe AS are referred to SAVR less frequently than male patients, ${ }^{8}$ though they present with more clinically advanced valve disease $e^{3,9-11}$ and worse valve hemodynamics $^{5,9-18}$ (- Tables 1, 2). There are several possible reasons for these findings. First, ventricular adaptation to a stenotic aortic valve is different in female patients, compared with male patients, with predominant concentric left ventricular (LV) hypertrophy rather than LV dilation. This may be the result of different hormonal, mostly estrogen, effects. ${ }^{19-22}$ Second, female patients with AS generally have better LV systolic function. $3,5,9,10,13-16,18,23,24$ Third, female patients have lower prevalence of cardiovascular comorbidities (such as coronary artery disease ${ }^{5,13,15,16,18,23-25}$ ) and as such are less likely to be referred to a routine echocardiogram, which otherwise may allow for an earlier diagnosis of aortic valve pathology, possibly while still asymptomatic. Moreover, studies examining aortic valve disease management have shown referral delays both when measuring time to diagnosis and time to surgery in female patients compared with male patients. ${ }^{8,26,27}$

Another important consideration of the heart team when determining on patient's appropriate valve management is the estimated surgical risk. Several studies have shown sex-related differences in outcome after cardiac surgery, ${ }^{28-30}$ though mainly focusing on coronary artery surgery. As a result, female sex has been considered an independent risk factor for perioperative mortality. ${ }^{31}$ Moreover, it has been shown that female patients with severe AS have a distinctive risk profile compared with male patients ( - Table 1 ). A female patient with severe AS is more likely to be older $3,5,11,13,14,18,23,32$ and with lower body surface area $3,5,13,16,24$ compared with male patients. Also, the diagnosis of frailty and porcelain aorta were 


\begin{tabular}{|c|c|c|c|c|c|c|c|c|c|c|c|}
\hline$\tilde{\omega} \tilde{\Delta}$ & $\begin{array}{l}0 \\
0 \\
+1 \\
\infty \\
0 \\
0 \\
1 \\
\sigma \\
\sigma\end{array}$ & 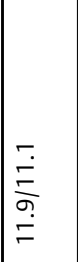 & $\frac{\alpha}{z}$ & $\begin{array}{l}\text { in } \\
\text { in } \\
+1 \\
m \\
\infty \\
\infty \\
1 n \\
+1 \\
0 \\
0 \\
0\end{array}$ & $\frac{o}{z}$ & $\frac{o}{z}$ & 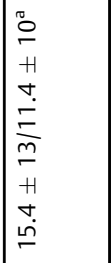 & $\begin{array}{l}\sigma \\
\sigma \\
+1 \\
\infty \\
\frac{\infty}{n n} \\
+1 \\
\infty \\
\sigma \\
\sigma\end{array}$ & 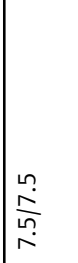 & 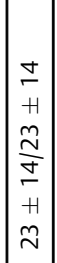 & $\frac{o}{z}$ \\
\hline 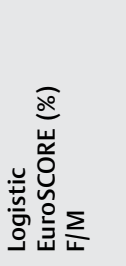 & $\frac{o}{z}$ & 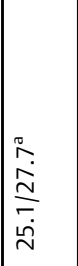 & 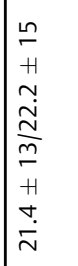 & 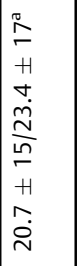 & 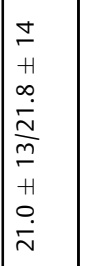 & 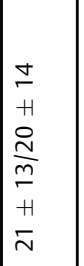 & 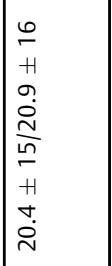 & $\frac{o}{z}$ & $\frac{o}{z}$ & $\frac{q}{z}$ & 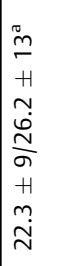 \\
\hline 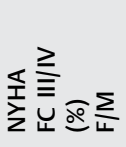 & 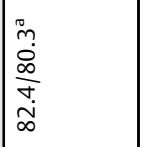 & $\frac{c}{z}$ & $\frac{o}{z}$ & 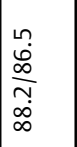 & 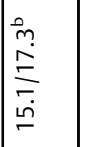 & 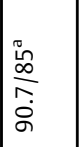 & $\begin{array}{l}\infty \\
\substack{\infty \\
\\
\infty}\end{array}$ & $\frac{o}{z}$ & $\mid \begin{array}{l}0 \\
\dot{\infty} \\
\infty \\
\\
\\
\infty \\
\infty\end{array}$ & 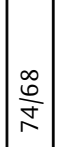 & $\begin{array}{l}\stackrel{a}{1} \\
\frac{\infty}{m} \\
\dot{m} \\
\dot{\infty}\end{array}$ \\
\hline 守す结 & 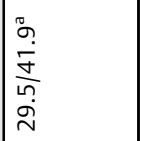 & 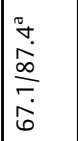 & 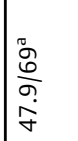 & $\begin{array}{l}\frac{1}{1} \\
\infty \\
\infty \\
0 \\
0 \\
\dot{0} \\
\dot{1}\end{array}$ & $\frac{a}{z}$ & $\begin{array}{l}\frac{0}{5} \\
\frac{5}{5} \\
\frac{5}{6} \\
\frac{1}{n}\end{array}$ & $\frac{x}{z}$ & $\frac{\mathscr{c}}{z}$ & 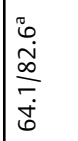 & 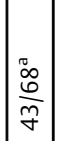 & $\begin{array}{l}\bar{\sigma} \\
\frac{\sigma}{\sigma} \\
\frac{\sigma}{\sigma} \\
\infty \\
\dot{\sigma}\end{array}$ \\
\hline 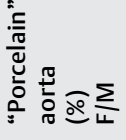 & $\begin{array}{l}0 \\
\stackrel{0}{0} \\
\frac{0}{r} \\
\end{array}$ & $\frac{o}{z}$ & $\frac{a}{z}$ & $\frac{o}{z}$ & $\frac{o}{z}$ & $\frac{o}{z}$ & $\frac{o}{z}$ & $\frac{o}{z}$ & & $\underset{\Xi}{\equiv}$ & $\frac{o r}{z}$ \\
\hline 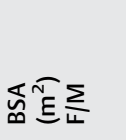 & 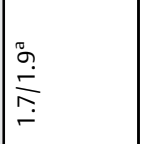 & 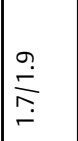 & $\frac{o}{z}$ & $\frac{o}{z}$ & 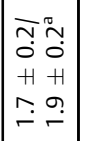 & $\frac{o}{z}$ & $\frac{a}{z}$ & $\frac{o}{z}$ & 竎 & 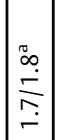 & 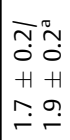 \\
\hline 突 & 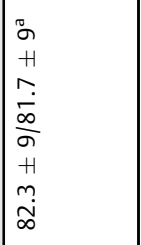 & $\mid \begin{array}{l}\bar{j} \\
\dot{d} \\
\frac{d}{\sigma} \\
\dot{\infty} \\
\infty\end{array}$ & $\begin{array}{l}\infty \\
\infty \\
+1 \\
0 \\
0 \\
\dot{\infty} \\
\frac{\infty}{\lambda} \\
+1 \\
+1 \\
\infty \\
\infty\end{array}$ & 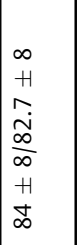 & $\begin{array}{l}0 \\
\infty \\
+1 \\
0 \\
\infty \\
0 \\
\infty \\
\frac{1}{\lambda} \\
+1 \\
0 \\
0 \\
\infty \\
\infty\end{array}$ & $\begin{array}{l}0 \\
0 \\
+1 \\
\tilde{1} \\
0 \\
\infty \\
0 \\
0 \\
+1 \\
\infty \\
\tilde{D} \\
\infty\end{array}$ & $\begin{array}{l}0 \\
\infty \\
+1 \\
+1 \\
0 \\
0 \\
\infty \\
\frac{1}{\lambda} \\
+1 \\
+1 \\
i \\
\infty \\
\infty\end{array}$ & $\begin{array}{l}\infty \\
\infty \\
+1 \\
+ \\
i \\
\infty \\
\infty \\
\infty \\
+1 \\
\omega n \\
\infty \\
\infty \\
\infty\end{array}$ & $\frac{\infty}{\infty}$ & 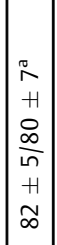 & 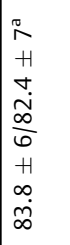 \\
\hline$\sum_{u} \frac{\widehat{d}}{=}$ & 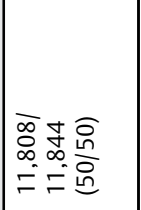 & 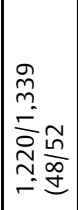 & 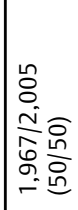 & 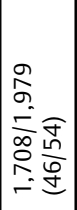 & 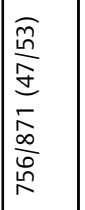 & 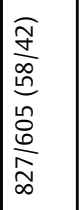 & 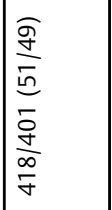 & 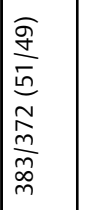 & 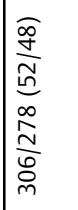 & 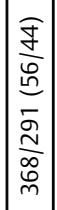 & 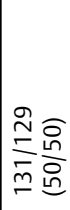 \\
\hline 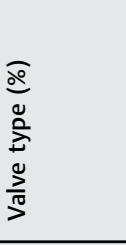 & 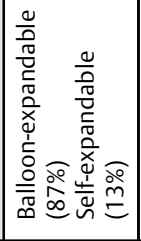 & $\begin{array}{l}\frac{\bar{v}}{n} \\
\stackrel{\frac{v}{v}}{n}\end{array}$ & 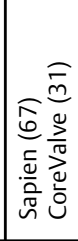 & 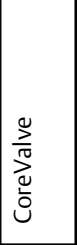 & 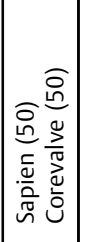 & 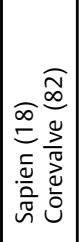 & 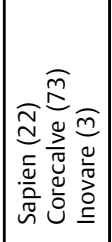 & 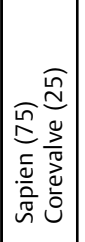 & 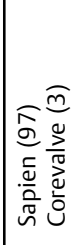 & 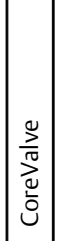 & 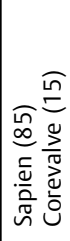 \\
\hline 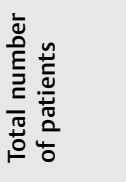 & \begin{tabular}{|l}
$\tilde{\hat{\sigma}}$ \\
$\tilde{\sim}$ \\
$\tilde{\sim}$
\end{tabular} & 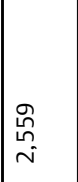 & $\mid \begin{array}{l}\tilde{N} \\
\tilde{m}\end{array}$ & $\mid \begin{array}{l}\infty \\
\infty \\
0 \\
m \\
-1\end{array}$ & $\begin{array}{l}\hat{a} \\
6 \\
-\end{array}$ & 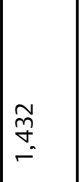 & $\frac{9}{\infty}$ & $\stackrel{\llcorner}{\wedge}$ & F & 曷 & $\stackrel{\stackrel{Q}{N}}{\sim}$ \\
\hline 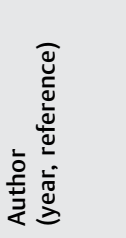 & 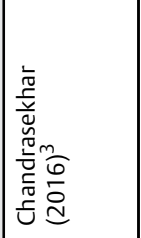 & 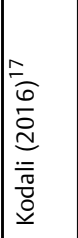 & 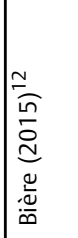 & 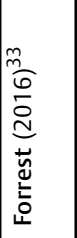 & 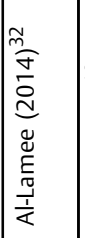 & 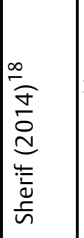 & 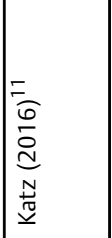 & 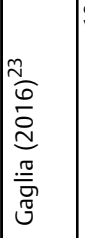 & 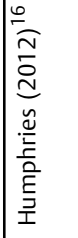 & 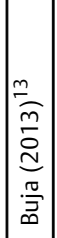 & 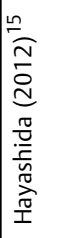 \\
\hline
\end{tabular}

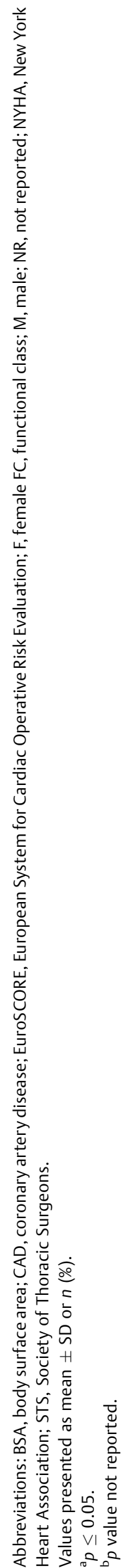


Table 2 Preprocedural echocardiographic parameters

\begin{tabular}{|c|c|c|c|c|c|c|c|}
\hline Author & $\begin{array}{l}\text { Total } \\
\text { number } \\
\text { of patients }\end{array}$ & $\begin{array}{l}\text { Female/Male } \\
n(\%)\end{array}$ & $\begin{array}{l}\text { LV systolic } \\
\text { EF }<30 \% \\
(\%)\end{array}$ & $\begin{array}{l}\text { Mean TV } \\
\text { gradient } \\
\text { (mm Hg) }\end{array}$ & $\begin{array}{l}\text { Maximal TV } \\
\text { gradient } \\
\text { (mm Hg) }\end{array}$ & $\begin{array}{l}\text { Aortic valve } \\
\text { area }\left(\mathrm{cm}^{2}\right)\end{array}$ & $\begin{array}{l}\text { Aortic annulus } \\
\text { diameter } \\
\text { (mm) }\end{array}$ \\
\hline $\begin{array}{l}\text { Chandrasekhar } \\
(2016)^{3}\end{array}$ & 23,652 & $\begin{array}{l}11,808 / 11,844 \\
(50 / 50)\end{array}$ & $4.3 / 9.6^{\mathrm{b}}$ & NR & NR & NR & NR \\
\hline $\begin{array}{l}\text { Kodali } \\
(2016)^{17}\end{array}$ & 2,559 & $\begin{array}{l}1,220 / 1,339 \\
(48 / 52)\end{array}$ & $\mathrm{NR}$ & $46.1 / 42^{a}$ & NR & $0.61 / 0.68^{a}$ & $18.3 / 19.9^{\mathrm{a}}$ \\
\hline $\begin{array}{l}\text { Bière } \\
(2015)^{12}\end{array}$ & 3,972 & $\begin{array}{l}1,967 / 2,005 \\
(50 / 50)\end{array}$ & NR & $\begin{array}{l}51.0 \pm 18 / \\
45.4 \pm 15^{\mathrm{a}}\end{array}$ & NR & NR & NR \\
\hline $\begin{array}{l}\text { Forrest } \\
(2016)^{33}\end{array}$ & 3,687 & $\begin{array}{l}1,708 / 1,979 \\
(46 / 54)\end{array}$ & NR & NR & NR & NR & NR \\
\hline $\begin{array}{l}\text { Al-Lamee } \\
(2014)^{32}\end{array}$ & 1,627 & $\begin{array}{l}756 / 871 \\
(47 / 53) \\
\end{array}$ & NR & NR & $\begin{array}{l}85 \pm 28 / \\
77 \pm 24 \\
\end{array}$ & $\begin{array}{l}0.6 \pm 0.2 / \\
0.7 \pm 0.5 \\
\end{array}$ & $\begin{array}{l}21.3 \pm 21 \\
23.2 \pm 2 \\
\end{array}$ \\
\hline $\begin{array}{l}\text { Sherif } \\
(2014)^{18}\end{array}$ & 1,432 & $\begin{array}{l}827 / 605 \\
(58 / 42)\end{array}$ & $8.5 / 16.4^{a}$ & $\begin{array}{l}52.1 \pm 21 / \\
45.5 \pm 16^{\mathrm{a}}\end{array}$ & $\begin{array}{l}46.7 \pm 41 / \\
41.9 \pm 36^{\mathrm{a}}\end{array}$ & $\begin{array}{l}0.7 \pm 0.4 / \\
0.72 \pm 0.4^{\mathrm{a}}\end{array}$ & NR \\
\hline $\begin{array}{l}\text { Katz } \\
(2016)^{11}\end{array}$ & 819 & $\begin{array}{l}418 / 401 \\
(51 / 49)\end{array}$ & NR & $\begin{array}{l}52.2 \pm 16 / \\
46.3 / 16^{\mathrm{a}}\end{array}$ & NR & $\begin{array}{l}0.6 \pm 0.21 \\
0.7 \pm 0.2^{a}\end{array}$ & NR \\
\hline $\begin{array}{l}\text { Gaglia } \\
(2016)^{23}\end{array}$ & 755 & $\begin{array}{l}383 / 372 \\
(51 / 49) \\
\end{array}$ & $\begin{array}{l}(\mathrm{EF}<40 \%) \\
16.2 / 30.1^{\mathrm{a}} \\
\end{array}$ & $\begin{array}{l}50.1 \pm 13 / \\
44.9 \pm 12^{\mathrm{a}}\end{array}$ & $\begin{array}{l}73.7 \pm 19 / \\
67.8 \pm 14^{\mathrm{a}} \\
\end{array}$ & $\begin{array}{l}0.63 \pm 0.1 / \\
0.70 \pm 0.1^{\mathrm{a}}\end{array}$ & NR \\
\hline $\begin{array}{l}\text { Humphries } \\
(2012)^{16}\end{array}$ & 502 & $\begin{array}{l}306 / 278 \\
(52 / 48)\end{array}$ & $12 / 28^{a}$ & $41 / 40^{a}$ & NR & $0.6 / 0.7^{\mathrm{a}}$ & NR \\
\hline $\begin{array}{l}\text { Buja } \\
(2013)^{13}\end{array}$ & 659 & $\begin{array}{l}368 / 291 \\
(56 / 44)\end{array}$ & $\begin{array}{l}(\mathrm{EF}<40 \%) \\
16 / 27^{\mathrm{a}}\end{array}$ & $\begin{array}{l}55 \pm 18 / \\
47 \pm 14^{\mathrm{a}}\end{array}$ & $\begin{array}{l}88 \pm 26 / \\
77 \pm 22^{\mathrm{a}}\end{array}$ & NR & $\begin{array}{l}21 \pm 2 / \\
23 \pm 2^{\mathrm{a}}\end{array}$ \\
\hline $\begin{array}{l}\text { Hayashida } \\
(2012)^{15}\end{array}$ & 260 & $\begin{array}{l}131 / 129 \\
(50 / 50)\end{array}$ & $\begin{array}{l}(\mathrm{EF}<40 \%) \\
19.8 / 39.5^{\mathrm{a}}\end{array}$ & $\begin{array}{l}49.7 \pm 20 / \\
45.5 \pm 16\end{array}$ & NR & $\begin{array}{l}0.59 \pm 0.2 / \\
0.61 \pm 0.1\end{array}$ & $\begin{array}{l}20.9 \pm 1 / \\
22.9 \pm 2^{\mathrm{a}}\end{array}$ \\
\hline
\end{tabular}

Abbreviations: EF, ejection fraction; LV, left ventricular; NR, not reported; TV, transvalvular.

Values are presented as mean \pm SD or $n(\%)$ for female/male.

${ }^{a} p \leq 0.05$.

${ }^{\mathrm{b}} p$ value not reported.

reported more often in female patients. ${ }^{3,11,16,33}$ All these elements are considerable factors that contribute to a higher surgical risk assessment in female patients, thus favoring the decision to refrain from SAVR in female patients more than males.

Nevertheless, data surprisingly support better long-term survival for female patients compared with male patients after SAVR, ${ }^{9,34,35}$ suggesting lack of SAVR-specific risk assessment tools and raising the question of a possible selection bias between sexes. One possible limitation in the evaluation of female patients for SAVR is the calculated surgical risk scores. Studies comparing surgical risk scores between sexes have shown conflicting results,, ,13,16,17,24,25,33 highlighting the need for valve surgery-specific scores. Another limitation is the frailty assessment. In most reports frailty was defined upon "eyeballing," which is influenced by personal biases. Advanced age, female sex, and lower body mass index are commonly used in the subjective "eyeball" evaluation of frailty but were not associated with a higher frailty score when comprehensively evaluated; ${ }^{36}$ hence there is the need for using objective tools in the evaluation of frailty. ${ }^{25,36-38}$ One way or another, as the PARTNER trial was published and TAVR emerged as a noninferior alternative for SAVR, 1,39 many female patients with severe AS, previously managed conservatively, were increasingly referred to TAVR.
Could TAVR be a better solution for a female patient with severe symptomatic AS?

In a retrospective subanalysis of high-risk patients in the pivotal PARTNER trial, female patients had lower late mortality with TAVR versus SAVR ${ }^{40}$ Nevertheless, TAVR carries other possible complications with distinct procedurerelated characteristics and outcomes in female patients compared with male patients (-Table 3 ). Periprocedural, female patients suffer from significantly higher rates of vascular $^{3,5,6,11,12,16,17,32,33}$ and bleeding $5,7,11,12,16,17,33$ complications compared with male patients. As for device success, stroke risk, and 30-day mortality, studies have not shown uniform results, but most available data indicate similar outcome for both sexes. $3,5,13,16,17,24$ Importantly, female patients present with lower long-term mortality rate following TAVR compared with male patients. ${ }^{5-7,40}$

Several computed tomography (CT) studies suggest that differences between sexes regarding acute and long-term outcome potentially relate to anatomic vasculature variations, especially those concerning the aortic root and the peripheral vessels. ${ }^{41,42}$ Interestingly, it has been recently demonstrated that smaller aortic root dimensions reflect a sex-specific difference, which could not be fully explained by the smaller body size of female patients. ${ }^{43}$ In contrast, peripheral vascular dimensions were not significantly different when adjusted 


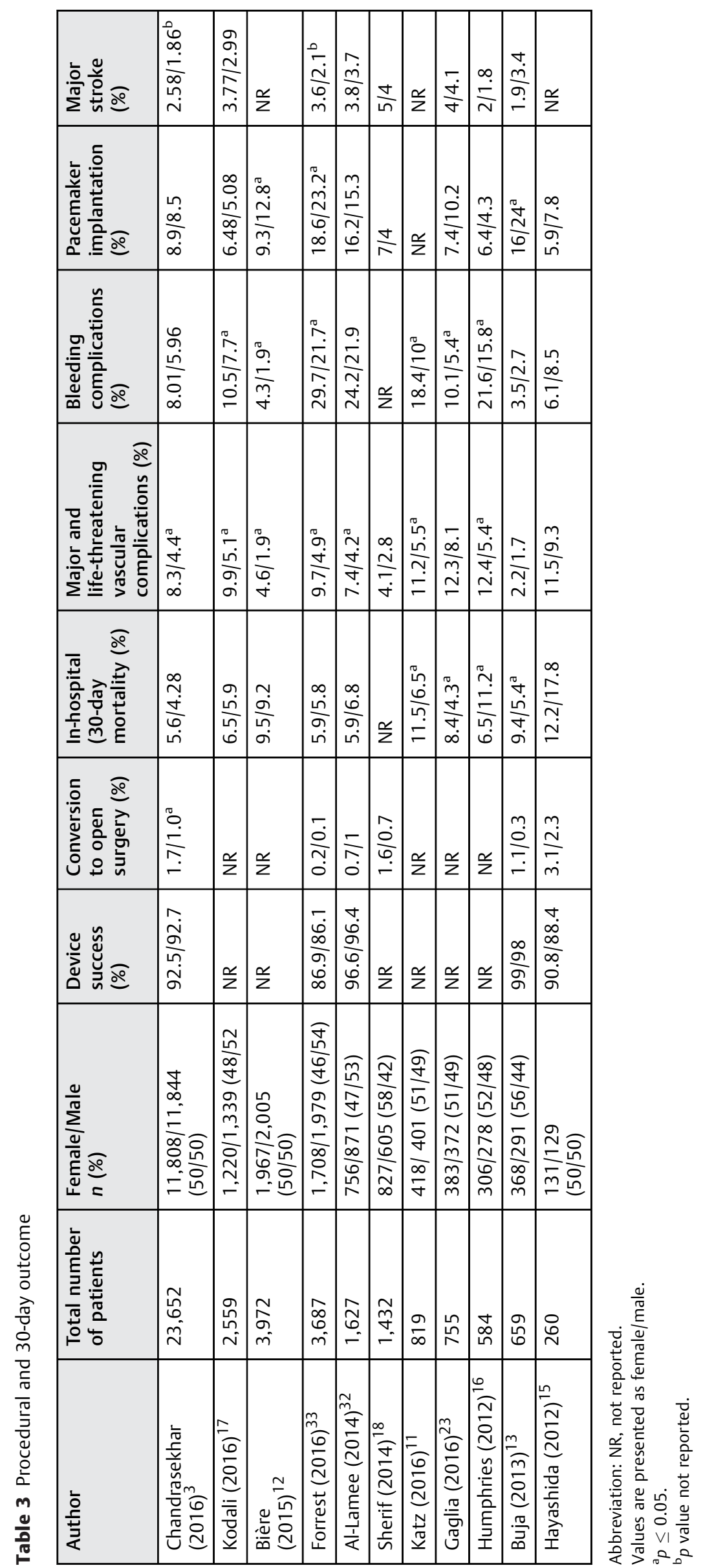


for body surface area. These sex-dependent anatomical differences have several implications on the TAVR procedure itself. First, due to their narrower aortic valve dimensions, ${ }^{5,11,16-18,23,24}$ significantly smaller valves are required for implantation in female patients. Hence, more female patients, compared with male patients, received balloon-expandable valves because of their availability in smaller diameters. ${ }^{3,11,12,18}$ Most likely related to this unequal distribution of device type, male patients are subject to more conduction abnormalities and thus to greater pacemaker insertion rates than female patients. ${ }^{5,6,12,13,33}$ Also, the US nationwide TAVR registry (TVT registry) has reported increased conversion to open surgery in female patients $(1.7 \%$ vs $1 \%, p<0.001)$ due to various causes (ventricular rupture, aortic dissection, annulus rupture)-all associated with smaller anatomical geometry. ${ }^{3}$ Second, significantly higher rate of TAVR performed via alternative access and higher rate of vascular complications $^{3,5-7,16,17,33}$ are most likely related to the smaller peripheral vascular diameters $5,23,43$ that are characterized by sheath to femoral artery ratio $>1 .^{30,44}$

Overall, despite the sex-related procedural aspects and complication profile, long-term prognosis, as shown on different global registries, was consistently favorable in female TAVR patients ${ }^{5-7,17,40}$ compared with male patients (-Table 4). The largest TVT registry ${ }^{3}$ demonstrated higher survival in female patients over a 1-year follow-up. Similar trends were reported by others. ${ }^{5,12,15,18,40}$ Moreover, recent results from the German Transcatheter Aortic Valve Interventions Registry have shown that female sex has a protective effect on 5-year mortality rate following TAVR (hazard ratio $[\mathrm{HR}]=0.66$; 95\% confidence interval $[\mathrm{CI}]: 0.56-0.77) .{ }^{45}$ This may be the outcome of several contributing factors. Physiologically, data have shown earlier remodeling and LV hypertrophy regression after SAVR in female patients. ${ }^{46}$ These benefits may extend to female patients having TAVR. ${ }^{47}$ Another plausible reason may be that, as already shown, female patients generally present with fewer baseline comorbidities, including LV dysfunction, compared with male patients, which may ultimately affect survival. To be noted that in a recently published post hoc analysis, the survival benefit for female patients was only observed in patients with a preprocedural left ventricular ejection fraction (LVEF) $<50 \%{ }^{48}$ Furthermore, female patients have an overall longer life expectancy, which irrespectively may influence the overall survival advantage of female patients over male patients. As for vascular complications, it seems that long-term survival is not significantly affected by the increased rate of periprocedural vascular events in female patients. Moreover, the use of new-generation devices compatible with smaller sheaths and the growing expertise of the operators will probably diminish the risk for those complications as well.

All the aforementioned sex-related differences concerning TAVR have led to the establishment of the Women's INternational Transcatheter Aortic Valve Implantation (WIN-TAVI) real-world registry that was the first all-female multinational registry. ${ }^{49}$ Interestingly, one of the investigators' novel findings was that remote pregnancy acted as a predictor of 30-day primary safety endpoint. Surely, more data are needed regarding the effect of pregnancies on long-term mortality.

Since the indication for TAVR is expanding to intermediate-risk surgical patients, it is interesting to find out whether sex-related issues still act as major modifiers. Data from the pivotal studies of intermediate-risk TAVR (SURTAVI ${ }^{50}$ and PARTNER $2^{51}$ ) showed that female patients constitute $<50 \%$ of those studies populations, in contrast to the high-risk TAVR studies. Surprisingly, stratified analysis of this intermediate-risk cohort showed similar outcomes in both sexes (Szerlip et al. TCT 2016, Washington DC, presented as a poster, ${ }^{52}$ ). There are several plausible causes contributing to those findings. As intermediate- versus high-risk female patients are usually younger, they may "lose" their advantage in longevity. Another cause could be the increased availability of larger valve sizes, allowing male patients to progressively present with improved valve performance and better clinical outcome. To be noted that a possible future consideration in intermediate-risk female patients is the development of degenerated valve post-TAVR. The need for

Table 4 Postprocedural long-term outcome

\begin{tabular}{|l|l|l|l|}
\hline Author & $\begin{array}{l}\text { Total number } \\
\text { of patients }\end{array}$ & $\begin{array}{l}\text { Female/Male } \\
\boldsymbol{n}(\%)\end{array}$ & $\begin{array}{l}\text { Mortality at 1 year } \\
\text { (F/M, \%) }\end{array}$ \\
\hline Chandrasekhar $(2016)^{3}$ & 23,652 & $11,808 / 11,844(50 / 50)$ & $21.3 / 24.5^{\text {a }}$ \\
\hline Kodali $(2016)^{17}$ & 2,559 & $1,220 / 1,339(48 / 52)$ & $19 / 25.9^{\mathrm{b}}$ \\
\hline Bière $(2015)^{12}$ & 3972 & $1,967 / 2,005(50 / 50)$ & $19.3 / 23.7^{\text {a }}$ \\
\hline Forrest $(2016)^{33}$ & 3,687 & $1,708 / 1,979(46 / 54)$ & $21.3 / 24.1$ \\
\hline Al-Lamee $(2014)^{32}$ & 1.627 & $756 / 871(47 / 53)$ & $22.4 / 21.9$ \\
\hline Sherif $(2014)^{18}$ & 1,432 & $827 / 605(58 / 42)$ & $17.3 / 23.6^{\text {a }}$ \\
\hline Katz $(2016)^{11}$ & 819 & $418 / 401(51 / 49)$ & $2-y e a r$ mortality: \\
\hline Gaglia $(2016)^{23}$ & & $383 / 372(51 / 49)$ & $29.7 / 25.9$ \\
\hline Buja $(2013)^{13}$ & 755 & $368 / 291(56 / 44)$ & $20.6 / 21.5$ \\
\hline
\end{tabular}

${ }^{a} p \leq 0.05$.

${ }^{\mathrm{b}} p$ value not reported. 
valve-in-valve procedure will surely present a problem for female patients due to their already smaller aortic valve area.

In conclusion, further randomized control studies with long-term follow-up focusing on sex-related differences will allow physicians to improve the management of female patients with severe symptomatic AS and to further identify female subgroups that preferentially benefit from TAVR.

\section{Funding}

None.

\section{Conflict of Interest}

None.

\section{References}

1 Smith CR, Leon MB, Mack MJ, et al; PARTNER Trial Investigators. Transcatheter versus surgical aortic-valve replacement in highrisk patients. N Engl J Med 2011;364(23):2187-2198

2 Nishimura RA, Otto CM, Bonow RO, et al; American College of Cardiology; American College of Cardiology/American Heart Association; American Heart Association. 2014 AHA/ACC guideline for the management of patients with valvular heart disease: a report of the American College of Cardiology/American Heart Association Task Force on Practice Guidelines. J Thorac Cardiovasc Surg 2014;148(01):e1-e132

3 Chandrasekhar J, Dangas G, Yu J, et al; STS/ACC TVT Registry. Sexbased differences in outcomes with transcatheter aortic valve therapy: TVT Registry From 2011 to 2014. J Am Coll Cardiol 2016; 68(25):2733-2744

4 Bonow RO, Greenland P. Population-wide trends in aortic stenosis incidence and outcomes. Circulation 2015;131(11):969-971

5 O'Connor SA, Morice MC, Gilard M, et al. Revisiting sex equality with transcatheter aortic valve replacement outcomes: a collaborative, patient-level meta-analysis of 11,310 patients. J Am Coll Cardiol 2015;66(03):221-228

6 Stangl V, Baldenhofer G, Laule M, Baumann G, Stangl K. Influence of sex on outcome following transcatheter aortic valve implantation (TAVI): systematic review and meta-analysis. J Interv Cardiol 2014;27(06):531-539

7 Zhao ZG, Liao YB, Peng Y, et al. Sex-related differences in outcomes after transcatheter aortic valve implantation: a systematic review and meta-analysis. Circ Cardiovasc Interv 2013;6(05):543-551

8 Bach DS, Radeva JI, Birnbaum HG, Fournier AA, Tuttle EG. Prevalence, referral patterns, testing, and surgery in aortic valve disease: leaving women and elderly patients behind? J Heart Valve Dis 2007;16(04):362-369

9 Fuchs C, Mascherbauer J, Rosenhek R, et al. Gender differences in clinical presentation and surgical outcome of aortic stenosis. Heart 2010;96(07):539-545

10 Duncan AI, Lin J, Koch CG, Gillinov AM, Xu M, Starr NJ. The impact of gender on in-hospital mortality and morbidity after isolated aortic valve replacement. Anesth Analg 2006;103(04):800-808

11 Katz M, Carlos Bacelar Nunes Filho A, Caixeta A, et al; Brazilian TAVI Registry investigators. Gender-related differences on short- and longterm outcomes of patients undergoing transcatheter aortic valve implantation. Catheter Cardiovasc Interv 2017;89(03):429-436

12 Bière L, Launay M, Pinaud $F$, et al. Influence of sex on mortality and perioperative outcomes in patients undergoing TAVR: insights from the FRANCE 2 registry. J Am Coll Cardiol 2015;65(07):755-757

13 Buja P, Napodano M, Tamburino C, et al; Italian Multicenter CoreValve Registry Investigators. Comparison of variables in men versus women undergoing transcatheter aortic valve implantation for severe aortic stenosis (from Italian Multicenter CoreValve registry). Am J Cardiol 2013;111(01):88-93
14 Conrotto F, D’Ascenzo F, Presbitero P, et al. Effect of gender after transcatheter aortic valve implantation: a meta-analysis. Ann Thorac Surg 2015;99(03):809-816

15 Hayashida K, Morice MC, Chevalier B, et al. Sex-related differences in clinical presentation and outcome of transcatheter aortic valve implantation for severe aortic stenosis. J Am Coll Cardiol 2012;59 (06):566-571

16 Humphries KH, Toggweiler S, Rodés-Cabau J, et al. Sex differences in mortality after transcatheter aortic valve replacement for severe aortic stenosis. J Am Coll Cardiol 2012;60(10):882-886

17 Kodali S, Williams MR, Doshi D, et al. Sex-specific differences at presentation and outcomes among patients undergoing transcatheter aortic valve replacement: a cohort study. Ann Intern Med 2016;164(06):377-384

18 Sherif MA, Zahn R, Gerckens U, et al. Effect of gender differences on 1-year mortality after transcatheter aortic valve implantation for severe aortic stenosis: results from a multicenter real-world registry. Clin Res Cardiol 2014;103(08):613-620

19 Villari B, Campbell SE, Schneider J, Vassalli G, Chiariello M, Hess OM. Sex-dependent differences in left ventricular function and structure in chronic pressure overload. Eur Heart J 1995;16(10): 1410-1419

20 Rohde LE, Zhi G, Aranki SF, Beckel NE, Lee RT, Reimold SC. Genderassociated differences in left ventricular geometry in patients with aortic valve disease and effect of distinct overload subsets. Am J Cardiol 1997;80(04):475-480

21 Piro M, Della Bona R, Abbate A, Biasucci LM, Crea F. Sex-related differences in myocardial remodeling. J Am Coll Cardiol 2010;55 (11):1057-1065

22 Aurigemma GP, Gaasch WH. Gender differences in older patients with pressure-overload hypertrophy of the left ventricle. Cardiology 1995;86(04):310-317

23 Gaglia MA Jr, Lipinski MJ, Torguson R, et al. Comparison in men versus women of co-morbidities, complications, and outcomes after transcatheter aortic valve implantation for severe aortic stenosis. Am J Cardiol 2016;118(11):1692-1697

24 Stangl V, Baldenhofer G, Knebel F, et al. Impact of gender on threemonth outcome and left ventricular remodeling after transfemoral transcatheter aortic valve implantation. Am J Cardiol 2012; 110(06):884-890

25 Chandrasekhar J, Mehran R. Poor mobility independently predicts mortality in TAVI: are we closer to a universal definition for frailty? Catheter Cardiovasc Interv 2015;86(07):1278-1279

26 Arber S, McKinlay J, Adams A, Marceau L, Link C, O'Donnell A. Patient characteristics and inequalities in doctors' diagnostic and management strategies relating to CHD: a video-simulation experiment. Soc Sci Med 2006;62(01):103-115

27 Raine RA, Black NA, Bowker TJ, Wood DA. Gender differences in the management and outcome of patients with acute coronary artery disease. J Epidemiol Community Health 2002;56(10): 791-797

28 Fox AA, Nussmeier NA. Does gender influence the likelihood or types of complications following cardiac surgery? Semin Cardiothorac Vasc Anesth 2004;8(04):283-295

29 Guru V, Fremes SE, Austin PC, Blackstone EH, Tu JV. Gender differences in outcomes after hospital discharge from coronary artery bypass grafting. Circulation 2006;113(04):507-516

30 Vaccarino V, Koch CG. Long-term benefits of coronary bypass surgery: are the gains for women less than for men? J Thorac Cardiovasc Surg 2003;126(06):1707-1711

31 Ad N, Barnett SD, Speir AM. The performance of the EuroSCORE and the Society of Thoracic Surgeons mortality risk score: the gender factor. Interact Cardiovasc Thorac Surg 2007;6(02):192-195

32 Al-Lamee R, Broyd C, Parker J, et al. Influence of gender on clinical outcomes following transcatheter aortic valve implantation from the UK transcatheter aortic valve implantation registry and the National Institute for Cardiovascular Outcomes Research. Am J Cardiol 2014;113(03):522-528 
33 Forrest JK, Adams DH, Popma JJ, et al. Transcatheter aortic valve replacement in women versus men (from the US CoreValve Trials). Am J Cardiol 2016;118(03):396-402

34 Kulik A, Lam BK, Rubens FD, et al. Gender differences in the longterm outcomes after valve replacement surgery. Heart 2009;95 (04):318-326

35 Rahimtoola SH. Valvular heart disease/cardiac surgery. J Am Coll Cardiol 2006;47(11, Suppl):D37-D40

36 Green P, Woglom AE, Genereux P, et al. The impact of frailty status on survival after transcatheter aortic valve replacement in older adults with severe aortic stenosis: a single-center experience. JACC Cardiovasc Interv 2012;5(09):974-981

37 Cockburn J, Singh MS, Rafi NH, et al. Poor mobility predicts adverse outcome better than other frailty indices in patients undergoing transcatheter aortic valve implantation. Catheter Cardiovasc Interv 2015;86(07):1271-1277

38 Rodés-Cabau J, Mok M. Working toward a frailty index in transcatheter aortic valve replacement: a major move away from the “eyeball test.". JACC Cardiovasc Interv 2012;5(09):982-983

39 Leon MB, Smith CR, Mack M, et al; PARTNER Trial Investigators. Transcatheter aortic-valve implantation for aortic stenosis in patients who cannot undergo surgery. N Engl J Med 2010;363 (17):1597-1607

40 Williams M, Kodali SK, Hahn RT, et al. Sex-related differences in outcomes after transcatheter or surgical aortic valve replacement in patients with severe aortic stenosis: insights from the PARTNER Trial (Placement of Aortic Transcatheter Valve). J Am Coll Cardiol 2014;63(15):1522-1528

41 Ferrante G, Pagnotta P, Petronio AS, et al. Sex differences in postprocedural aortic regurgitation and mid-term mortality after transcatheter aortic valve implantation. Catheter Cardiovasc Interv 2014;84(02):264-271

42 Naoum C, Blanke P, Dvir D, et al. Clinical outcomes and imaging findings in women undergoing TAVR. JACC Cardiovasc Imaging 2016;9(04):483-493
43 Hamdan A, Barbash I, Schwammenthal E, et al. Sex differences in aortic root and vascular anatomy in patients undergoing transcatheter aortic valve implantation: a computed-tomographic study. J Cardiovasc Comput Tomogr 2017;11(02):87-96

44 Hayashida K, Lefèvre T, Chevalier B, et al. Transfemoral aortic valve implantation new criteria to predict vascular complications. JACC Cardiovasc Interv 2011;4(08):851-858

45 Zahn R, Werner N, Gerckens U, et al; German Transcatheter Aortic Valve Interventions-Registry investigators. Five-year follow-up after transcatheter aortic valve implantation for symptomatic aortic stenosis. Heart 2017; heartjnl-2016-311004

46 Petrov G, Regitz-Zagrosek V, Lehmkuhl E, et al. Regression of myocardial hypertrophy after aortic valve replacement: faster in women? Circulation 2010;122(11, Suppl):S23-S28

47 Clavel MA, Webb JG, Rodés-Cabau J, et al. Comparison between transcatheter and surgical prosthetic valve implantation in patients with severe aortic stenosis and reduced left ventricular ejection fraction. Circulation 2010;122(19):1928-1936

48 Bauer T, Möllmann H, Beckmann A, et al. Left ventricular function determines the survival benefit for women over men after transcatheter aortic valve implantation (TAVI). EuroIntervention 2017;13(04):467-474

49 Chieffo A, Petronio AS, Mehilli J, et al; WIN-TAVI Investigators. Acute and 30-day outcomes in women after TAVR: results From the WIN-TAVI (Women's INternational Transcatheter Aortic Valve Implantation) real-world registry. JACC Cardiovasc Interv 2016;9 (15):1589-1600

50 Reardon MJ, Van Mieghem NM, Popma JJ, et al; SURTAVI Investigators. Surgical or transcatheter aortic-valve replacement in intermediate-risk patients. N Engl J Med 2017;376(14):1321-1331

51 Leon MB, Smith CR, Mack MJ, et al; PARTNER 2 Investigators. Transcatheter or surgical aortic-valve replacement in intermediate-risk patients. N Engl J Med 2016;374(17):1609-1620

52 Szerlip M. Transcatheter aortic valve replacement: only one of the advantages of being female.J Am Coll Cardiol 2016;68(25):2745-2746 